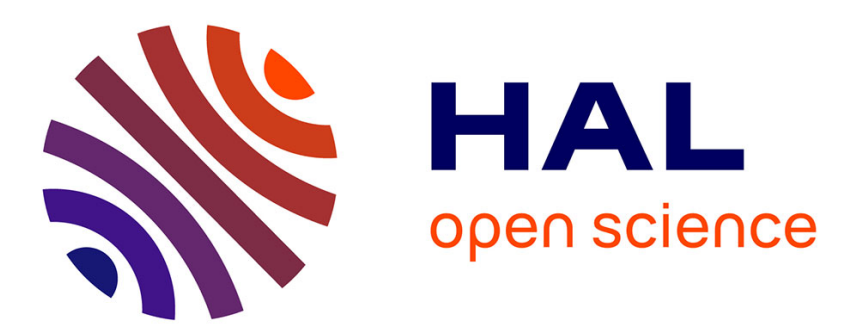

\title{
Complex amplitudes tracking loop for multi-path channel estimation in OFDM systems: Synthesis and extension
}

Huaqiang Shu, Laurent Ros, Eric Pierre Simon

\section{To cite this version:}

Huaqiang Shu, Laurent Ros, Eric Pierre Simon. Complex amplitudes tracking loop for multi-path channel estimation in OFDM systems: Synthesis and extension. ISCCSP 2014 - 6th International Symposium on Communications, Control and Signal Processing, May 2014, Athènes, Greece. 4 p. hal-01064688

\section{HAL Id: hal-01064688 \\ https://hal.science/hal-01064688}

Submitted on 16 Sep 2014

HAL is a multi-disciplinary open access archive for the deposit and dissemination of scientific research documents, whether they are published or not. The documents may come from teaching and research institutions in France or abroad, or from public or private research centers.
L'archive ouverte pluridisciplinaire HAL, est destinée au dépôt et à la diffusion de documents scientifiques de niveau recherche, publiés ou non, émanant des établissements d'enseignement et de recherche français ou étrangers, des laboratoires publics ou privés. 


\section{COMPLEX AMPLITUDES TRACKING LOOP FOR MULTI-PATH CHANNEL ESTIMATION IN OFDM SYSTEMS : SYNTHESIS AND EXTENSION (CORRECTED VERSION)}

\author{
Huaqiang Shu, Laurent Ros \\ GIPSA-Lab \\ Images and Signal Department \\ BP 46, 38402 Saint Martin d'Hères, France
}

\author{
Eric Pierre Simon \\ IEMN Lab \\ TELICE group \\ 59655 Villeneuve d'Ascq, France
}

\begin{abstract}
This study deals with pilot-aided multi-path channel estimation for orthogonal frequency division multiplexing (OFDM) systems under slow to moderate fading conditions. Some algorithms exploit the channel time-domain correlation by using Kalman filters (KFs) to track the channel multi-path complex amplitudes (CAs), assuming a primary acquisition of the delays. Recently, it was shown that less complex algorithms, based on a second-order Complex Amplitude Tracking Loop (CATL) structure and a Least-Square (LS) pilot-aided error signal, can also reach near optimal asymptotic mean-squared error (MSE) performance. The LS-CATL-based algorithms are inspired by digital Phase-Locked Loops (PLL), as well as by the "prediction-correction" principle of the KF (in steadystate mode). This paper sums up and extends our previous results for the tuning and steady-state performance of the LSCATL algorithm: analytic formulae are given for the first-, second-, and third-order loops, usable here for the multi-path multi-carrier scenario, and adaptable to any Doppler spectrum model of wide-sense stationary channels.
\end{abstract}

Index Terms - OFDM, Channel estimation, Kalman filter, Phase-locked loop

\section{INTRODUCTION}

Orthogonal frequency division multiplexing is an effective technique for alleviating frequency-selective channel effects in wireless communication systems. Reliable channel estimation in OFDM systems is crucial for coherent detection of the information symbols. Most of the conventional methods work in a symbol-by-symbol scheme [1]. More advanced algorithms exploit the time-domain correlation of the channel by using also past symbols. Various approaches are then possible as revisited in [2]: a natural way is to use Kalman Filters (KFs) to perform the joint estimation of the multi-path CAs. In this perspective, a linear recursive state-space model, such as the $r$ th order auto-regressive (AR $r$ ) model or the $r$ th order integrated Random-Walk ( $\mathrm{RW} r$ ) model [3], is used to approximate the channel CAs dynamic. In the literature, the most widely used model is certainly the AR1-model combined with a correlation-matching (CM) criterion to fix the AR1 coefficient [4]. This leads to the $\mathrm{AR} 1_{C M}-\mathrm{KF}$ algorithm (see many references and a review in [2]). Recently, the RW $r$-modelbased KFs [3] are proved to be more efficient, especially for the slow to moderate fading channels $\left(f_{d} T \leq 10^{-2}\right)$. However, the complexity of the RWr-KF for the joint estimation of the path CAs in OFDM remains high. We now focus on a reduced complexity adaptive algorithm, named here $\mathrm{RW} r$ LS-CATL, which can be interpreted as a simplification of the reference $\mathrm{RW} r-\mathrm{KF}$, for the same model order $r=1,2,3$. The RW2-LS-CATL algorithm $(r=2)$, was introduced in [5], theoretically analysed in [2], and shows almost the same asymptotic performance as a second-order KF algorithm. The RWrLS-CATL is inspired by digital PLLs and is based on a CATL structure, and on a specific CA error signal. This error signal is created from the LS estimate of the paths CA obtained from the pilot sub-carriers of the current OFDM symbol. The LSCATL presents a linear complexity in terms of the number of pilot-subcarriers $N_{p}$ versus a cubic complexity $\left(O\left(N_{p}^{3}\right)\right)$ for the reference Kalman algorithms (see [2]-table 1).

The key ideas to interpret the RWr-LS-CATL as a simplified RWr-KF stand in two main points: per-path processing, and steady-state mode. First, the front-end pilot-aided LS estimator makes it possible to convert the primary observation at pilot frequencies into a primary instantaneous estimate of the path CAs and then to obtain separate error signals for the paths. This preliminary estimation step allows at the output a per-path processing that filters each path CA separately, instead of a full joint processing in the RW $r$-KF. Secondly, the RWr-LS-CATL acts in each branch, i.e. for each multipath component, as a single-path single-carrier RW $r$-CATL [6], and then achieves the same asymptotic performance as a single-path single-carrier RWr-KF, but with a reduced complexity (due to the use of constant coefficients, instead of time-varying $\mathrm{KF}$ coefficients which require matrix inversion at each iteration). Indeed, [3] proves that, the $\mathrm{RW} r-\mathrm{KF}$ is equivalent in steady-state mode and slow-tracking scenario to the RWr-CATL with fixed (analytically derived) parameters. This point generalizes the connection (for the second-order) between PLL and KF to the third-order. 
The contribution of this paper is multi-fold as an extension of our previous studies that were restricted either to the second-order loops and the Rayleigh channel with Jakes' Doppler spectrum, or to the single-carrier single-path scenario. Here, we extend the RW-LS-CATL to the third order, and provide a synthesis about analytic formulae for the tuning and steady-state performance of the RW $r$-LS-CATL $(r=1,2,3)$, usable for the multi-path multi-carrier scenario and adaptable to any Doppler spectrum model of a wide-sense stationary channel. It is noteworthy that the $\mathrm{RW} r$-LS-CATL refers to the multi-path multi-carrier context whereas the $\mathrm{RW} r$-CATL refers to the single-path single-carrier context. This paper is organised as follows: section 2 presents the model. Then in section 3, we explain the RWr-LS-CATL, and give the tables containing analytic formulae for the tuning and its optimized performance. Finally, section 4 presents some simulation results and the conclusion.

Notations: $[\mathbf{x}]_{k}$ denotes the $k$ th entry of the vector $\mathbf{x}$, and $[\mathbf{X}]_{m, n}$ denotes the $[m, n]$ th entry of the matrix $\mathbf{X}$ (indices begin from 1). The notation $\operatorname{diag}\{\mathbf{x}\}$ is a diagonal matrix with $\mathbf{x}$ on its main diagonal. $\mathbf{I}_{N}$ is an $N \times N$ identity matrix. The symbols of $\{\cdot\}^{T}$ and $\{\cdot\}^{H}$ stand for the transpose and Hermitian operators respectively.

\section{OFDM SYSTEM}

Let us consider an OFDM system with $N$ subcarriers, and a cyclic prefix length $N_{g}$. The duration of an OFDM symbol is $T=N_{T} T_{s}$, where $T_{s}$ is the sampling time and $N_{T}=N+$ $N_{g}$. Let $\mathbf{x}_{(k)}$ be the sequence of transmitted elementary symbols of the $k$ th OFDM symbol. The $n$th element $\left[\mathbf{x}_{(k)}\right]_{n}(n=$ $1, \ldots, N)$ is phase modulated ( $M$-PSK) or quadrature amplitude modulation ( $M$-QAM) symbol that modulates the subcarrier with index $n-1-\frac{N}{2}$. The sequence of transmitted symbols is assumed to be zero-mean and stationary with normalized variance: $\mathrm{E}\left\{\left|\left[\mathbf{x}_{(k)}\right]_{n}\right|^{2}\right\}=1$. After transmission over a slowly time-varying (the channel is considered to be invariant during one OFDM symbol time $T$ ) multi-path channel and fast Fourier transform demodulation, the $k$ th received OFDM symbol $\mathbf{y}_{(k)}$ is given by:

$$
\mathbf{y}_{(k)}=\mathbf{H}_{(k)} \mathbf{x}_{(k)}+\mathbf{w}_{(k)},
$$

where $\mathbf{w}_{(k)}$ is an $N \times 1$ zero-mean complex circular Gaussian noise vector with covariance matrix $\sigma_{\mathrm{w}}^{2} \mathbf{I}_{N}$, and $\mathbf{H}_{(k)}$ is an $N \times$ $N$ diagonal matrix with its diagonal elements given by:

$$
\left[\mathbf{H}_{(k)}\right]_{n, n}=\frac{1}{N} \sum_{l=1}^{L}\left[\alpha_{(k)}^{(l)} \cdot e^{-j 2 \pi\left(\frac{n-1}{N}-\frac{1}{2}\right) \tau^{(l)}}\right],
$$

where $L$ is the total number of propagation paths, $\alpha_{(k)}^{(l)}$ is the CA of the $l$-th path at $k$ th OFDM symbol, and $\tau^{(l)} \times T_{s}$ is the $l$-th delay $\left(\tau^{(l)}\right.$ is not necessarily an integer, but $\tau^{(l)}<N_{g}$ ). The variable $\alpha_{(k)}^{(l)}$ is supposed to be a narrow-band stationary process, with a Power Spectrum Density (PSD) $\Gamma_{\alpha}(f)$ with a support limited within $\pm f_{d}$. The $L$ individual elements of $\left\{\alpha_{(k)}^{(l)}\right\}(l=1, \ldots, L)$ are uncorrelated with respect to one another, with global variance $\sigma_{\alpha}^{2}=\sum_{l=1}^{L} \sigma_{\alpha^{(l)}}^{2}$. Using (2), the observation model of (1) can be re-written [7] as:

$$
\mathbf{y}_{(k)}=\operatorname{diag}\left\{\mathbf{x}_{(k)}\right\} \mathbf{F} \boldsymbol{\alpha}_{(k)}+\mathbf{w}_{(k)},
$$

where $\boldsymbol{\alpha}_{(k)}=\left[\begin{array}{lll}\alpha_{(k)}^{(1)} & \ldots & \alpha_{(k)}^{(L)}\end{array}\right]^{T}$ and $\mathbf{F}$ is an $N \times L$ Fourier matrix depending on the delay distribution, with elements given by: $[\mathbf{F}]_{k, l}=e^{-j 2 \pi\left(\frac{k-1}{N}-\frac{1}{2}\right) \tau^{(l)}}$.

The $N_{p}$ pilot sub-carriers are evenly inserted into the $N$ sub-carriers at the positions $\mathcal{P}=\left\{n_{p} \mid n_{p}=(p-1) L_{f}+\right.$ $\left.1, p=1, \ldots, N_{p}\right\}$ with $L_{f}$ as the distance between two adjacent pilots. The received pilot sub-carriers can be written as:

$$
\mathbf{y}_{\mathbf{p}(k)}=\mathbf{X}_{(k)} \boldsymbol{\alpha}_{(k)}+\mathbf{w}_{\mathbf{p}(k)}
$$

with $\mathbf{X}_{(k)}=\operatorname{diag}\left\{\mathbf{x}_{\mathbf{p}(k)}\right\} \mathbf{F}_{\mathbf{p}}$. The $N_{p} \times 1$ vectors $\mathbf{x}_{\mathbf{p}}, \mathbf{y}_{\mathbf{p}}$ and $\mathbf{w}_{\mathbf{p}}$ correspond respectively to the sent data symbol, the received data symbol and the channel noise on the pilot subcarriers. The $N_{p} \times L$ matrix $\mathbf{F}_{\mathbf{p}}$ is the Fourier matrix for the pilot sub-carriers, with elements given by: $\left[\mathbf{F}_{\mathbf{p}}\right]_{n_{p}, l}=$ $e^{-j 2 \pi\left(\frac{n_{p}-1}{N}-\frac{1}{2}\right) \tau^{(l)}}$, where $n_{p} \in \mathcal{P}$.

\section{RW-LS-CATL AND ASYMPTOTIC MSE}

Inspired by a PLL structure, the RWr-CATL is a $r$-th order loop used for tracking the CAs of a single-path (flat-fading) channel in a single carrier system. The derivation of the CATL structure from the KF was carried out in [6] for the single-path single-carrier context. In the case of multi-path channel in a multi-carrier system, it can be extended to a vector-structured loop by employing an LS estimator at the front-end of the main loop [2]. The equations of the global LS-CATL algorithm are given below:

LS estimate:

$$
\hat{\boldsymbol{\alpha}}_{\mathbf{L s}(k)}=\mathbf{G}_{(k)} \mathbf{y}_{\mathbf{p}(k)},
$$

with $\mathbf{G}_{(k)}=\left(\mathbf{X}_{(k)}^{H} \mathbf{X}_{(k)}\right)^{-1} \mathbf{X}_{(k)}^{H}$ the LS operator.

Error signal: $\quad \boldsymbol{v}_{\boldsymbol{\epsilon}(k)}=\hat{\boldsymbol{\alpha}}_{\mathbf{L S}(k)}-\hat{\boldsymbol{\alpha}}_{(k \mid k-1)}$,

Loop filter:

$$
\begin{aligned}
& \boldsymbol{v}_{\mathrm{Lag} 1(k)}=\boldsymbol{v}_{\mathrm{Lag} 1(k-1)}+\boldsymbol{v}_{\boldsymbol{\epsilon}(k)}, \\
& \boldsymbol{v}_{\mathrm{Lag} 2(k)}=\boldsymbol{v}_{\mathrm{Lag} 2(k-1)}+\boldsymbol{v}_{\mathrm{Lag} 1(k)}, \\
& \boldsymbol{v}_{\mathbf{c}(k)}=\mu_{1} \boldsymbol{v}_{\boldsymbol{\epsilon}(k)}+\mu_{2} \boldsymbol{v}_{\mathbf{L a g} 1(k)}+\mu_{3} \boldsymbol{v}_{\mathbf{L a g} 2(k-1)},
\end{aligned}
$$

Numeric command generator:

$$
\begin{aligned}
\hat{\boldsymbol{\alpha}}_{(k+1 \mid k)} & =\hat{\boldsymbol{\alpha}}_{(k \mid k-1)}+\boldsymbol{v}_{\mathbf{c}(k)}, \\
\text { Final estimation: } \quad \hat{\boldsymbol{\alpha}}_{(k \mid k)} & =\hat{\boldsymbol{\alpha}}_{(k \mid k-1)}+\mu_{1} \boldsymbol{v}_{\boldsymbol{\epsilon}(k)} .
\end{aligned}
$$

The RW $r$-LS-CATL is in fact controlled by the loop filter coefficients $\left(\mu_{1}, \cdots, \mu_{r}\right)$. As a PLL-structured loop, the CATL can also be characterized by the physical parameters used for 


\begin{tabular}{cccc}
\hline \hline & RW1 & RW2 & RW3 \\
\hline Sub-optimal & $f_{c} T=\left(\frac{2 S_{\alpha}}{\pi \sigma_{\mathbf{L S}}^{2}}\right)^{\frac{1}{3}}$ & $\zeta=0.5$ & $m=3.19$ \\
parameters & & $f_{n} T=\left(\frac{2 S_{\alpha}}{\pi \sigma_{\mathbf{L S}}^{2}}\right)^{\frac{1}{5}}$ & $f_{n} T=\left(\frac{S_{\alpha} Q}{4 \pi \sigma_{\mathrm{LS}}^{2}}\right)^{\frac{1}{7}}$ \\
\hline$S_{\alpha}$ & $\int_{-\frac{1}{2 T}}^{+\frac{1}{2 T}} \Gamma_{\alpha}(f) \cdot(f T)^{2} d f$ & $\int_{-\frac{1}{2 T}}^{+\frac{1}{2 T}} \Gamma_{\alpha}(f) \cdot(f T)^{4} d f$ & $\int_{-\frac{1}{2 T}}^{+\frac{1}{2 T}} \Gamma_{\alpha}(f) \cdot(f T)^{6} d f$ \\
$\sigma_{\epsilon \alpha}^{2}$ & $\frac{S_{\alpha}}{\left(f_{c} T\right)^{2}}$ & $\frac{S_{\alpha}}{\left(f_{n} T\right)^{4}}$ & $\frac{S_{\alpha}}{(m \zeta)^{2}\left(f_{n} T\right)^{6}}$ \\
$\sigma_{\epsilon \mathrm{W}}^{2}$ & $\pi f_{c} T \sigma_{\mathrm{LS}}^{2}$ & $2 \pi f_{n} T \cdot\left(\zeta+\frac{1}{4 \zeta}\right) \sigma_{\mathrm{LS}}^{2}$ & $2 \pi f_{n} T \cdot \mathcal{B} \sigma_{\mathrm{LS}}^{2}$ \\
$C$ & $\frac{3}{4}(4 \pi)^{\frac{2}{3}}$ & $\frac{5}{4}(2 \sqrt{2} \pi)^{\frac{4}{5}}$ & $(2 \pi)^{\frac{6}{7}}\left[\frac{1}{(m \zeta)^{2}} \cdot\left(\frac{2}{Q}\right)^{\frac{6}{7}}+\mathcal{B} \cdot\left(\frac{Q}{2}\right)^{\frac{1}{7}}\right]$ \\
$\sigma_{\epsilon \min }^{2}$ & $C\left(\sigma_{\mathbf{L S}}^{2}\right)^{\frac{2}{3}}\left(S_{\alpha}\right)^{\frac{1}{3}}$ & $C\left(\sigma_{\mathbf{L S}}^{2}\right)^{\frac{4}{5}}\left(S_{\alpha}\right)^{\frac{1}{5}}$ & $C\left(\sigma_{\mathbf{L S}}^{2}\right)^{\frac{6}{7}}\left(S_{\alpha}\right)^{\frac{1}{7}}$ \\
\hline \hline
\end{tabular}

Table 1. Asymptotic expressions for the RW $r$-LS-CATL (under the assumption $f_{d} T<<1$ )

the PLLs of the same order. For RW3, the links between $\left(\mu_{1}\right.$, $\left.\mu_{2}, \mu_{3}\right)$ and the capacitance ratio $m$, the damping factor $\zeta$ and the natural frequency $f_{n}$ were found in [6]; for RW2, $\mu_{3}=0$, the relations between $\left(\mu_{1}, \mu_{2}\right)$ and $\left(\zeta, f_{n}\right)$ are given in [2]; for RW1, $\mu_{2}=\mu_{3}=0$, the relation between $\mu_{1}$ and the cut-off frequency $f_{c}$ could also be found in [2].

By using (4) and the definition of $\mathbf{G}_{(k)}$, we have:

$$
\hat{\boldsymbol{\alpha}}_{\mathbf{L S}(k)}=\boldsymbol{\alpha}_{(k)}+\mathbf{w}_{\mathbf{L S}(k)},
$$

with $\mathbf{w}_{\mathbf{L S}(k)}$ the input noise of the loop:

$$
\mathbf{w}_{\mathbf{L S}(k)}=\mathbf{G}_{(k)} \mathbf{w}_{\mathbf{p}(k)} .
$$

The covariance matrix of $\mathbf{w}_{\mathbf{L S}(k)}$ is: $E\left\{\mathbf{w}_{\mathbf{L S}(k)} \cdot \mathbf{w}_{\mathbf{L S}(k)}^{H}\right\}=$ $\sigma_{\mathrm{w}}^{2} \cdot\left(\mathbf{F}_{\mathbf{p}}^{H} \mathbf{F}_{\mathbf{p}}\right)^{-1}$. The average variance per-path can then be calculated as:

$$
\sigma_{\mathrm{LS}}^{2}=\frac{1}{L} \cdot \sigma_{\mathrm{w}}^{2} \cdot \operatorname{Trace}\left\{\left(\mathbf{F}_{\mathbf{p}}^{H} \mathbf{F}_{\mathbf{p}}\right)^{-1}\right\} .
$$

Note that, $\mathbf{F}_{\mathbf{p}}^{H} \mathbf{F}_{\mathbf{p}}$ is in general not a diagonal matrix, except for the special case where the path delays of channel $\tau^{(l)} T_{s}$ are multiples of the sampling time $T_{s}$, i.e., if $\tau^{(l)}$ are integers.

According to (6), the error signal $\boldsymbol{v}_{\boldsymbol{\epsilon}(k)}$ is actually the difference between a noisy observation of the CA vector $\hat{\alpha}_{\mathbf{L s}(k)}$ (see (12)) and last prediction $\boldsymbol{\alpha}_{(k \mid k-1)}$. The role of LS estimator is to produce a preliminary estimate of $\boldsymbol{\alpha}$ from the pilot sub-carriers of the current OFDM symbol. Such an error signal remains co-linear with the prediction error $\boldsymbol{\alpha}_{(k)}-$ $\hat{\boldsymbol{\alpha}}_{(k \mid k-1)}$, and is free from inter-path interference. Thus the $\mathrm{RW} r$-LS-CATL can be interpreted as a parallel structure that is in fact a combination of $L$ single-path single-carrier $\mathrm{RW} r$ CATLs (in (6)-(11)), with the LS estimate of CA to the input of the loop. Consequently, the analysis of the RW $r$-LS-CATL can be done directly by exploiting the results of the corresponding RW $r$-CATL. In the single-path single-carrier scenarios, the asymptotic expressions of the RW3-CATL were given in our previous work [6], these results can be applied to every branch (each one corresponds to a single path) of the RW3-LS-CATL by using the loop noise variance $\sigma_{\mathrm{LS}}^{2}$ instead of the observation noise variance $\sigma_{\mathrm{w}}^{2}$. This was also deduced for the lower-order loops $(r=1,2)$ in [2].

We aim to find the asymptotic MSE (mean value per path) defined by : $\sigma_{\epsilon}^{2}=\frac{1}{L} \cdot E\left\{\left(\boldsymbol{\alpha}_{(k)}-\hat{\boldsymbol{\alpha}}_{(k)}\right)^{H} \cdot\left(\boldsymbol{\alpha}_{(k)}-\hat{\boldsymbol{\alpha}}_{(k)}\right)\right\}$. As explained in [2], $\sigma_{\epsilon}^{2}$ is comprised of two parts:

$$
\sigma_{\epsilon}^{2}=\sigma_{\epsilon \alpha}^{2}+\sigma_{\epsilon \mathrm{W}}^{2},
$$

where $\sigma_{\epsilon \alpha}^{2}$, the dynamic error variance, results from the highpass filtering of the input CA $\alpha_{(k)}$ and $\sigma_{\epsilon \mathrm{W}}^{2}$, the static error variance, is the low-pass filtering of the input loop noise.

The closed-form results for the RW $r$-LS-CATL $(r=$ $1,2,3)$ are synthesized in table 1 . In this table, the coefficients $\mathcal{B}$ and $Q$ are functions of the sub-optimal parameters $m$ and $\zeta$, with detailed expressions given in [6]. By using $m=3.19$ and $\zeta=0.39$, we have $\mathcal{B}=1.72$ and $Q=4.5$. The value of $S_{\alpha}$ can be calculated for a given channel Doppler spectrum $\Gamma_{\alpha}(f)$. Besides of the widely used Jakes' Doppler spectrum (used in our previous works), one can also choose a 3D channel model with a constant Doppler spectrum [8] for the indoor scenarios. In both cases, the integration term $S_{\alpha}$ can be found in closed-form expression $S_{\alpha}=S \cdot\left(f_{d} T\right)^{2 r} \cdot \frac{\sigma_{\alpha}^{2}}{L}$, where $S$ is a constant with respect to the chosen spectrum and loop order. As an example for the 3D model, $S=1 /(2 r+1)=1 / 3,1 / 5,1 / 7$ respectively for $r=1,2,3$. From the summary table, we find that the asymptotic minimum MSE of the RW $r$-LS-CATL $\sigma_{\epsilon}^{2}$ is proportional to the $\frac{2 r}{2 r+1}$ power of the path loop noise variance $\sigma_{\mathrm{LS}}^{2}$, and is also proportional to the $\frac{2 r}{2 r+1}$ power of $f_{d} T$, the detailed computations can be found in [9]. 


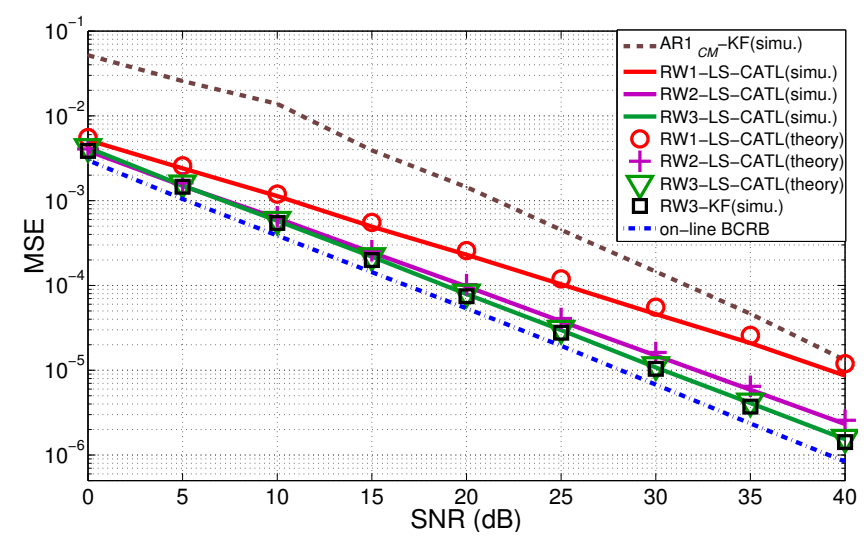

Fig. 1. The MSE versus the SNR with $f_{d} T=10^{-3}, N_{p}=16$

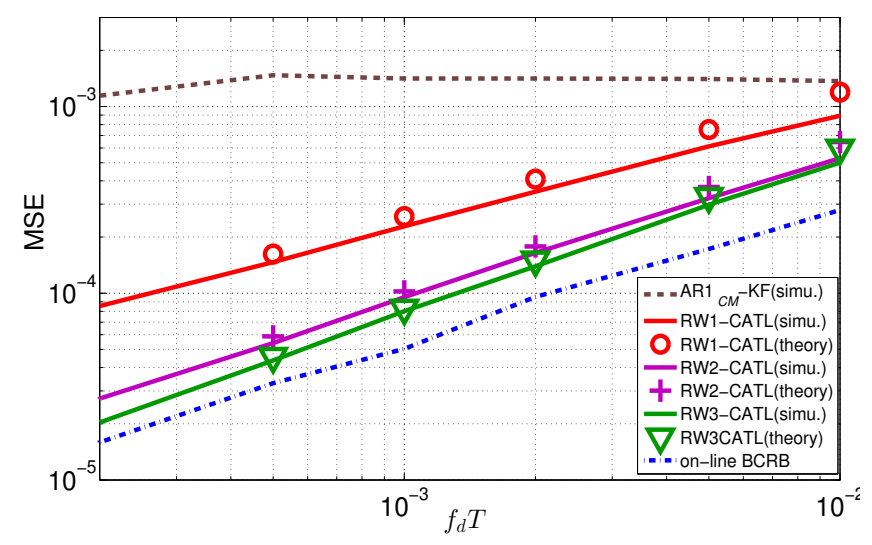

Fig. 2. The MSE versus $f_{d} T$ with $\mathrm{SNR}=20 \mathrm{~dB}, N_{p}=16$

\section{SIMULATION AND CONCLUSION}

To validate the proposed approximate method and the analytic results, we simulate a 4QAM-OFDM system with $N=128$ sub-carriers. By default, the OFDM system has $N_{g}=16$ samples of $\mathrm{CP}, N_{p}=16$ pilot sub-carriers in each transmitted OFDM symbol and the system bandwidth is $1 / T_{s}=2 \mathrm{MHz}$. The GSM 6-tap outdoor Rayleigh channel model was chosen as the simulation channel (as used in [2]).

Figure 1 shows the simulated and theoretical asymptotic MSE (mean value per path) of the RW-LS-CATLs, as a function of the SNR for $f_{d} T=10^{-3}$. Also, We plot as a reference the BCRB (Bayesian Cramer-Rao lower bound) [2], and the simulated performance of the $\mathrm{AR} 1_{C M}-\mathrm{KF}$ algorithm. Figure 2 presents the MSE evolution as a function of $f_{d} T$. We can clearly see that the RW-LS-CATLs outperform the $\mathrm{AR} 1_{C M}$-KF. Moreover, the MSEs obtained by the simulation approximately coincide with the theoretical values, and are more accurate when $f_{d} T$ decreases (note that we have made approximations under low $f_{d} T$ assumption for the analytical MSE formulae). As argued in the previous section, for $r=1,2,3$, the asymptotic MSE are respectively proportional to the $2 / 3,4 / 5,6 / 7$ powers of the $f_{d} T$, and are inversely pro- portional to the $2 / 3,4 / 5,6 / 7$ powers of the SNR. Additionally, we attach the simulation results of a numerically optimized RW3-KF in figure 1 to show that the simplified RW3-LSCATL can almost reach the asymptotic performance of the RW3-KF. This corroborates the results of [2] obtained for the 2nd-order loop.

In conclusion, we have considered the RW $r$-LS-CATL as a per-path processing approach to track the multi-path channel CAs in a multi-carrier context. The analytic optimization of the first three order loops are synthesized and generalized for any given Doppler spectrum of the channel. Simulation results validate our theoretical analysis, and show that the RW-LS-CATL can asymptotically outperforms the AR1model-based Kalman filter (under CM criterion) and reach the RW-KF performance.

\section{ACKNOWLEDGMENT}

We thank the french ANR CORRIDOR project for his financial support.

\section{REFERENCES}

[1] S. Coleri, M. Ergen, A. Puri, and A. Bahai, "Channel estimation techniques based on pilot arrangement in OFDM systems," IEEE Trans. Broadcast., vol. 48, no. 3, pp. 223-229, 2002.

[2] L. Ros, H. Hijazi, and E. P. Simon, "Complex amplitudes tracking loop for multipath channel estimation in OFDM systems under slow to moderate fading," ELSEVIER Signal Process., vol. 97, pp. 134-145, 2014.

[3] H. Shu, E. P. Simon, and L. Ros, "Third-order kalman filter: Tuning and steady-state performance," IEEE Signal Process. Lett., vol. 20, no. 11, pp. 1082-1085, 2013.

[4] W. Chen and R. Zhang, "Kalman-filter channel estimator for OFDM systems in time and frequency-selective fading environment," in Proc. IEEE Intl. Conf. Acoust. Speech, Signal Process., 2004, vol. 4, pp. 377-380.

[5] L. Ros, H. Hijazi, and E. P. Simon, "Paths complex gain tracking algorithms for ofdm receiver in slowly-varying channels," in Proc. Intl. Symp. Commun. Control, Signal Process., Mar. 2010, pp. 1-6.

[6] H. Shu, L. Ros, and E. P. Simon, "Third-order Complex Amplitudes Tracking Loop for Slow to Moderate Flat Fading Channel On-Line Estimation,” IET commun., 2014.

[7] B. Yang, K. B. Letaief, R. S. Cheng, and Z. Cao, "Channel estimation for OFDM transmission in multipath fading channels based on parametric channel modeling," IEEE Trans. Commun., vol. 49, no. 3, pp. 467-479, 2001.

[8] R. H. Clarke and W. L. Khoo, "3-D mobile radio channel statistics,” IEEE Trans. Veh. Technol., vol. 46, no. 3, pp. 798 -799, aug 1997.

[9] H. Shu, Algorithmes de poursuite pour l'estimation de canal radio-mobile et performances asymptotiques: applications pour les systèmes OFDM (accessible on line), Ph.D. thesis, Université Lille 1, Sciences et Technologies, Nov. 2013. 